\title{
Stability Properties of POD-Galerkin Approximations for the Compressible Navier-Stokes Equations
}

Angelo Iollo - Stéphane Lanteri — Jean-Antoine Désidéri

$$
\mathbf{N}^{\circ} 3589
$$

Decembre 1998

THÈME 4 



\title{
Stability Properties of POD-Galerkin Approximations for the Compressible Navier-Stokes Equations
}

\author{
Angelo Iollo, Stéphane Lanteri, Jean-Antoine Désidéri \\ Thème 4 - Simulation et optimisation \\ de systèmes complexes \\ Projet Sinus
}

Rapport de recherche $\mathrm{n}^{\circ} 3589$ - Decembre 1998 - 27 pages

\begin{abstract}
Fluid flows are very often governed by the dynamics of a small number of coherent structures, i.e., fluid features which keep their individuality during the evolution of the flow. The purpose of this paper is to study a low order simulation of the NavierStokes equations on the basis of the evolution of such coherent structures. One way to extract from flow simulations some basis functions which can be interpreted as coherent structures is by Proper Orthogonal Decomposition (POD). Then, by means of a Galerkin projection, it is possible to find the system of ODEs which approximates the problem in the finite dimensional space spanned by the POD basis functions. It is found that the low order modeling of relatively complex flow simulations, such as the laminar vortex shedding from an airfoil at incidence and the turbulent vortex shedding from a square cylinder, provides good qualitative results compared with reference computations. In this respect, it is shown that the accuracy of numerical schemes based on simple Galerkin projection is insufficient and numerical stabilization is needed. To conclude, we approach the issue of the optimal selection of the norm used in the POD for the compressible Navier-Stokes equations by several numerical tests.
\end{abstract}

Key-words: POD, Navier-Stokes equations, Galerkin method, low order model 


\section{Proprietés de stabilité des approximations de Galerkin basées sur la POD - Application aux équations des Navier-Stokes compressibles}

Résumé : Les écoulements de fluides sont souvent gouvernés par la dynamique d'un petit nombre de structures cohérentes, c'est-à-dire par des caractéristiques physiques qui existent tout au long de l'évolution de l'écoulement. L'objectif de cet article est d'étudier une méthode de simulation d'ordre faible des équations de Navier-Stokes sur la base de l'évolution de telles structures. Une manière d'extraire de simulations d'écoulements des fonctions de base qui peuvent être interprétées comme des structures cohérentes est par la technique de Décompositon Orthogonale en Modes Propres ( POD » pour « Proper Ortogonal Decomposition »). Ensuite, en appliquant une méthode de projection de Galerkin, il est possible d'exhiber le système d'équations différentielles oridinaires (EDO) qui approchent le problème

dans l'espace de dimension finie engendré par les fonctions de base POD. On observe alors que la modélisation d'ordre faible de simulations d'écoulements relativement complexes, tels que l'écoulement laminaire autour d'un profil d'aile en forte incidence et l'écoulement turbulent autour d'un cylindre carré (qui représentent deux situations caractérisées par un détachement périodique de tourbillons en amont de l'écoulement), fournit de bons résultats qualitatifs comparativement à ceux résultant de simulations classiques de référence. Dans cet article, nous démontrons que la précision de schémas numériques basés sur une projection de Galerkin simple est insuffisante et qu'il est nécessaire d'introduire une stabilisation numérique. Pour conclure, nous menons une étude préliminaire sur la sélèction d'une norme appropriée pour la POD appliquée aux équations de Navier-Stokes pour un fluide compressible, sur la base de plusieurs expérimentations numériques.

Mots-clés : POD, équations de Navier-Stokes, methode de Galerkin, modél d'ordre faible 


\section{Introduction}

The natural ambition of the aerodynamic designer is to influence the structure of the flow (for example by local injection of fluid) with the aim of increasing or optimizing performance. For this reason automatic shape design and active flow control are object of intense theoretical and practical research. One central problem in applications is the prohibitive amount of computational resources needed by repeated application of a flow solver, in particular in the case of three-dimensional, unsteady (possibly turbulent) flows. A promising technique to circumvent this difficulty is the adoption of low order models as governing equations. Such models should provide a qualitative description of the main features of the flow, for example in terms of lift and drag versus time, while permitting very economical numerical solution.

One way to devise a low order model is to use the Proper Orthogonal Decomposition (POD), by which it is possible to extract from a database of direct simulations a certain number of basis functions onto which the Navier-Stokes equations are projected. The NavierStokes equations are thus reduced to a finite-dimensional system of nonlinear ODEs. If the dimension of this system is reasonably small, the solution can be found with very limited computational effort.

The POD was introduced in fluid mechanics by Lumley [1] in the study of turbulent flows (for a review see [2]). In that setting, the basis functions obtained by POD were recognized to be coherent structures, i.e. spatial features which repeatedly appear in space and time. In those works, the basic assumption was that a limited number of coherent structures capture most of the dynamics of the system, relying on the fact that turbulence might be the manifestation in the physical space of a strange attractor of limited dimension in phase space. We retain in the following the same assumption, i.e., that a limited number of coherent structures (about 50 in our cases) suffices to describe the flow. In fact, this is verified a posteriori.

It may be objected that such an approach is of limited applicability since the basis functions obtained for a given configuration or parameter regime are not suitable for other simulations. Typically this is the case when the control is acting on the flow, so that new coherent structures, not present in the uncontrolled flow, appear. Although this is true in general, there are many examples in which the coherent structures do not qualitatively change for wide ranges of the flow parameters. In addition, there are techniques to enlarge the computational database in order to include the effect of controls. For example, it is proposed in [5] to simulate the flow under the action of a random control to generate a sufficiently rich database, which could also encompass the coherent structures of the specific control subsequently used.

In this study we contribute in two ways to the construction of a robust scheme for the integration of the system of ODEs resulting from the projection of the compressible NavierStokes equations. Firstly, on the basis of what was done in [7] for the Euler equations, we show by numerical evidence that when a low order model is constructed using POD to describe the main flow features, it is not necessary to model the unresolved modes in some alternate way to achieve numerical stability. Aubry et al. [4] presented a model for the flat plate turbulent boundary layer based on a Galerkin projection of the Navier-Stokes

$\mathrm{RR} \quad \mathrm{n}^{\circ} 3589$ 
equations onto POD eigenfunctions resulting from experiments. The unresolved modes have been accounted for by a model for the small scales which ensures the proper amount of dissipation (and stabilization), so that the system of ordinary differential equations obtained is stable. In our perspective, POD can only enlarge the stability domain of the numerical scheme in the complex plane. This is found by classical numerical analysis tools for a onedimensional advection-diffusion equation and verified by some numerical experiments for an unsteady laminar flow, in which a textbook four-stage Runge-Kutta time integration scheme advances with a CFL number of 75 ! We conclude that numerical stabilization of POD-Galerkin approximation of the Navier-Stokes equations can be attained by a judicious use of numerical dissipation as an alternative to modeling the unresolved modes as was proposed in [4].

The second contribution of this work is the discussion concerning the canonical $L^{2}$ norm in the definition of POD. For example, to retain the optimality of the POD basis functions, we show that in the simple case of the linear advection-diffusion equation with Dirichlet conditions, it is preferable to include a weighting function in the definition of the scalar product. In the case of the turbulent Navier-Stokes equations, a numerical test involving POD basis functions obtained using the $H^{1}$ formulation, showed that the resulting low order model is more dissipative and hence less subject to numerical instability than the regular $L^{2}$ formulation.

We anticipate that a grater sophistication in the devise employed for numerical stability will ultimately contribute to apply optimal flow control techniques to the compressible Navier-Stokes equations as successfully as in the incompressible case, for which more results have yet been established in the literature.

The article is organized as follows. In Section 2, POD basis functions are determined in the setting of Hilbert-Schmidt theory of kernels. In Section 3, we analyze the POD approximation of the 1D advection-diffusion with different boundary conditions. We show that POD can be interpreted as a filter and we compare the constant coefficients system of ODEs with the POD filtering. In the subsequent Section 4, we present the numerical experimentation setting and the results obtained with the POD filter for the case of the flow past a NACA0012 at $20^{\circ}$ of incidence, Mach $=0.2, R e=2100$ (laminar flow) and for the flow about a square cylinder, Mach $=0.1, R e=22000$ (turbulent flow). For the same cases we present the results obtained with the $H^{1}$ POD filter. To conclude, the results obtained with the constant coefficients system of ODEs are compared with those obtained by POD filtering.

\section{POD of the compressible Navier-Stokes equations}

The method of "snapshots" proposed by Sirovich [3] for the incompressible Navier-Stokes equations is adapted here to the compressible Navier-Stokes equations. This method, briefly outlined below for completeness, allows for the construction of the basis functions starting from a limited number of "snapshots" of the flow at successive times. 
Consider the non-dimensional Navier-Stokes equations in two dimensions written in conservative form with $W=\{\rho, \rho u, \rho v, \rho e\}$

$$
\begin{gathered}
W_{t}+F_{x}+G_{y}=\frac{1}{R e}\left(R_{x}+S_{y}\right) \\
F=\left\{\rho u, \rho u^{2}+p, \rho u v, u(\rho e+p)\right\} \text { and } G=\left\{\rho v, \rho u v, \rho v^{2}+p, v(\rho e+p)\right\}
\end{gathered}
$$

denote the convective fluxes while

$$
R=\left\{0, \frac{4}{3} u_{x}-\frac{2}{3} v_{y}, v_{x}+u_{y}, u\left(\frac{4}{3} u_{x}-\frac{2}{3} v_{y}\right)+v\left(v_{y}+u_{x}\right)+\frac{\gamma}{\gamma-1} \frac{T_{x}}{\operatorname{Pr}}\right\}
$$

and

$$
S=\left\{0, v_{x}+u_{y}, \frac{4}{3} v_{y}-\frac{2}{3} u_{x}, v\left(\frac{4}{3} v_{y}-\frac{2}{3} u_{x}\right)+u\left(v_{y}+u_{x}\right)+\frac{\gamma}{\gamma-1} \frac{T_{y}}{P r}\right\}
$$

denote the diffusive fluxes. In the above expressions $\rho$ is the density, $u$ and $v$ are the cartesian components of the flow speed, $e$ is the total energy per unit mass and $T$ is the temperature. The pressure is given by $p=1 / 2(\gamma-1)\left(2 \rho e-\rho u^{2}-\rho v^{2}\right)$ and $\gamma$ is the specific heat ratio.

Suppose that from existing data, the solution at $M$ different time steps $t_{n}$ is known in terms of $W^{(n)}=W\left(x, y ; t_{n}\right)$. It is required to find a function

$$
\phi(x)=\left(\phi_{1}, \phi_{2}, \phi_{3}, \phi_{4}\right) \in\left(L^{2}\left(R^{2}\right)\right)^{4}
$$

which gives the best representation of the set of $W^{(n)}$ in the following sense

$$
\frac{<\left(W^{(n)}, \phi\right)^{2}>}{(\phi, \phi)}=\max _{\psi} \frac{<\left(W^{(n)}, \psi\right)^{2}>}{(\psi, \psi)}
$$

where $(\psi, \psi)$ denotes the canonical $L^{2}$ inner product and the brackets $<\cdot>$ indicate the time average

$$
<\left(W^{(n)}, \psi\right)^{2}>=\frac{1}{M} \sum_{n=1}^{M}\left(W^{(n)}, \psi\right)^{2}
$$

In other words, one seeks a function $\phi$ which is most parallel (correlated) in an average sense to the given solution set $W^{(n)}$.

This problem finds its natural setting in the theory of linear integral equations (see chapter III of [6]). Here some results relevant to our study are sketched in the frame of such a theory. For omitted proofs we refer to [6].

Consider first the general quadratic integral form

$$
J(\psi, \psi)=\int_{\Omega} \psi(s) K(s, t) \psi(t) d t d s
$$

where $K(s, t)=K(t, s)$ is a symmetric $4 \times 4$ matrix and $\psi \in\left(L^{2}\left(R^{2}\right)\right)^{4}$. Suppose that the functional $J$ takes on positive values only, i.e. $\forall \psi: J(\psi, \psi)>0$; in this case $J(\psi, \psi)$ is said to be positive definite. Suppose also that $(\psi, \psi)=1$. 
If the function $\phi^{(l)}$ satisfies

$$
\int_{\Omega} K(s, t) \phi^{(l)}(t) d t=\lambda_{l} \phi^{(l)}(s)
$$

then clearly $J\left(\phi^{(l)}, \phi^{(l)}\right)=\lambda_{l}$, and it can be shown that $\phi^{(l)}(s)$ realizes a local maximum of $J(\psi, \psi)$ subject to the constraint $(\psi, \psi)=1$.

Now, with the particular choice of the kernel

$$
K(s, t)=\frac{1}{M} \sum_{n=1}^{M} W^{(n)}(s) \otimes W^{(n)}(t)
$$

where $\otimes$ is the tensorial product, we have

$$
J(\psi, \psi)=\frac{1}{M} \sum_{n=1}^{M}\left[\int_{\Omega} \psi(s) W^{(n)}(s) d s \int_{\Omega} \psi(t) W^{(n)}(t) d t\right]=<\left(W^{(n)}, \psi\right)^{2}>
$$

Therefore the maximum of $J(\psi, \psi)$ with $(\psi, \psi)=1$ solves the problem in eq. 2 . Substituting eq. 6 into eq. 5 we have

$$
\frac{1}{M} \sum_{n=1}^{M} \int_{\Omega} W^{(n)}(s) \otimes W^{(n)}(t) \phi^{(l)}(t) d t=\lambda_{l} \phi^{(l)}(s)
$$

This is an eigenvalue problem for the symmetric, degenerate, positive definite kernel in eq. 6 . In this respect, recall that every continuous symmetric kernel that does not vanish identically possesses eigenvalues and eigenfunctions. Also since the kernel in eq. 8 is real, symmetric and degenerate then the eigenvalues are $M$ in number and all real. The eigenvalues are positive since $J(\psi, \psi)$ in eq. 7 is positive definite.

The totality of eigenvalues and eigenfunctions of eq. 8 are found sequentially. Each new eigenfunction is sought as the solution of the maximization problem for the functional $J$ subject to the constraint of being orthogonal to all previously found eigenfunctions. Any $r$-fold degenerate eigenvalue is associated with $r$ linearly independent eigenfunctions.

In conclusion, once the eigenvalue problem of eq. 8 is solved, we are left with a set of $M$ eigenvalues $\lambda_{n}$ and $M$ orthonormal eigenfunctions $\phi^{(n)}$ which give an optimal representation, in the sense made precise at the beginning of this section, of the solution known at different time steps. In the next Sections we use similar arguments to determine a basis for the approximation of the compressible Navier-Stokes equations.

Sirovich [3] proposed an efficient and simple way to solve the eigenvalue problem based on the fact, that the eigenfunctions can be expressed in terms of the original set of data, i.e.

$$
\phi(s)=\sum_{n=1}^{M} \chi_{n} W^{(n)}(s)
$$


where the $\chi_{n}(n=1 \ldots M)$ are scalar coefficients. Substituting eq. 9 in eq. 8 results in a linear algebra problem consisting of finding the eigenvalues and the eigenvectors of

$$
C \mathcal{K}=\lambda \mathcal{K}
$$

where $\mathcal{K}=\left(\chi_{1}, \ldots, \chi_{M}\right)$ is one of the $M$ eigenvectors, $\lambda$ is the corresponding eigenvalue, and $C=1 / M\left(W^{(i)}, W^{(j)}\right)$ for $i, j=1, \ldots, M$. In the applications of the following Sections we make use of the POD-functions gradients, which we compute simply by

$$
\nabla \phi(s)=\sum_{n=1}^{M} \chi_{n} \nabla W^{(n)}(s)
$$

When the solution $W$ is sampled by means of a large number of snapshots, a certain number of eigenvalues are very likely to be close to zero so that the contribution of the corresponding eigenfunction to the description of the flow may be considered negligible. In this case we can consider only the first $M_{c}$ (normally about 50) eigenvalues for the projection of the governing equations over the corresponding eigenfunctions, in order to reduce the set of ODEs to be solved. In practical applications, we normally solve this eigenproblem by the use of NAG library routines.

Before considering applications to flow simulations, we analyze in the next section general properties of the POD approach when applied to single model problems representative of flow equations.

\section{Analysis of POD applied to model equations}

Let us consider first the periodic solutions of the pure advection equation

$$
u_{t}+u_{x}=0
$$

with $x \in[0,2 \pi]$. A semi-discretization over the uniform mesh $x_{i}=i 2 \pi / N(i=0, \ldots, N)$ results in the following set of ODEs ("method of lines")

$$
U_{t}+\frac{N}{2 \pi} A U=0
$$

where $U=\left\{u\left(x_{i}\right)\right\}$ and $A=\left\{a_{n i}\right\}$ is the spatial discretization operator. In this case, the POD basis functions are (unsurprisingly) the usual Fourier modes

$$
\phi^{k}\left(x_{i}\right)=\frac{1}{\sqrt{N}} \exp [\iota k 2 \pi i / N]
$$

so that, substituting in eq. 12 the expression

$$
U=\sum_{k=-M}^{M} w^{k}(t)\left\{\phi^{k}\left(x_{i}\right)\right\}
$$

$\mathrm{RR} \mathrm{n}^{\circ} 3589$ 
and making the $l^{2}$ (discrete) projection over the mode $\bar{\phi}^{j}$ yield the following reduced set of ODEs

$$
w_{t}^{j}+\sum_{k=-M}^{M} w^{k}\left\{\bar{\phi}^{j}\left(x_{n}\right)\right\}\left\{a_{n i}\right\}\left\{\phi^{k}\left(x_{i}\right)\right\}=0 \quad j=1, \ldots, M
$$

The matrix $\Lambda=\left\{\lambda_{j k}\right\}=\left\{\left\{\bar{\phi}^{j}\left(x_{n}\right)\right\}\left\{a_{n i}\right\}\left\{\phi^{k}\left(x_{i}\right)\right\}\right\}$ is diagonal as the $\left\{\phi^{j}\left(x_{i}\right)\right\}$ are its eigenvectors. On the diagonal of $\Lambda$ appear the eigenvalues of $A$, the discrete operator.

From eq. 14, multiplying by $\phi^{j}$ and subsequently summing over $j$, we reconstruct the $\mathrm{PDE}$ in the physical space. We obtain

$$
U_{t}+\frac{N}{2 \pi} \widetilde{A} A U=0
$$

with $\widetilde{A}=\left\{\tilde{a}_{n i}\right\}=\left\{\sum_{k=-M}^{M} \bar{\phi}^{k}\left(x_{n}\right) \phi^{k}\left(x_{i}\right)\right\}$. The matrix $\widetilde{A}$, which is circulant and symmetric, has the same eigenvectors as matrix $A$. Its first $M$ eigenvalues (not to be confused with the eigenvalues of the correlation matrix which yields the POD basis functions) are 1 , whereas the remaining are 0 . Therefore, the first $M$ eigenvalues of the spatial operator $\widetilde{A} A$ are equal to those of $A$, the others are canceled out.

For example, suppose that the spatial operator is the usual upwind differentiation, and suppose that a Runge-Kutta scheme is used to integrate eq. 15 forward in time. Since the higher frequencies are filtered out by the matrix $\widetilde{A}$, the maximum CFL allowed is higher, see fig. 1. In this figure the eigenvalues of the upwind spatial operator are plotted in the complex plane. They lie over a circle $\mathcal{C}$ of radius 1 tangent to the imaginary axis, the lower frequencies corresponding to the eigenvalues closer to the origin. Suppose we map $\mathcal{C}$ onto a circle $\mathcal{C}^{\prime}$ which is tangent to the domain of stability of the time-integration scheme, e.g. the four stage Runge-Kutta scheme, by an homothetic transform. The radius of $\mathcal{C}^{\prime}$ is equal to the maximum allowed CFL number. Clearly, the smaller the number of retained frequencies, the higher the radius of $\mathcal{C}^{\prime}$. In the following Section we show that this is experimentally verified also in the case of the unsteady Navier-Stokes equations.

This simple analysis should clarify why the sole POD cannot be responsible for the stability degradation of the numerical scheme observed for example in [8]. On the contrary, the effect of the POD may be to enlarge the stability domain of the semidiscrete linear system. Also it is seen that POD can be viewed as a filter possibly adapted to the flow under consideration. We will use this interpretation to check if fairly complex flows can be conveniently represented by a reduced number of basis functions.

The same type of analysis can be applied with some cautions to other linear spatial operators with different boundary conditions. For example, if we consider the advectiondiffusion equation with homogeneous Dirichlet boundary conditions

$$
u_{t}+c u_{x}-\nu u_{x x}=0
$$

if central differencing is used

$$
A=\operatorname{Trid}\left(-\nu / h^{2}-c / 2 h, 2 \nu / h^{2},-\nu / h^{2}+c / 2 h\right)
$$




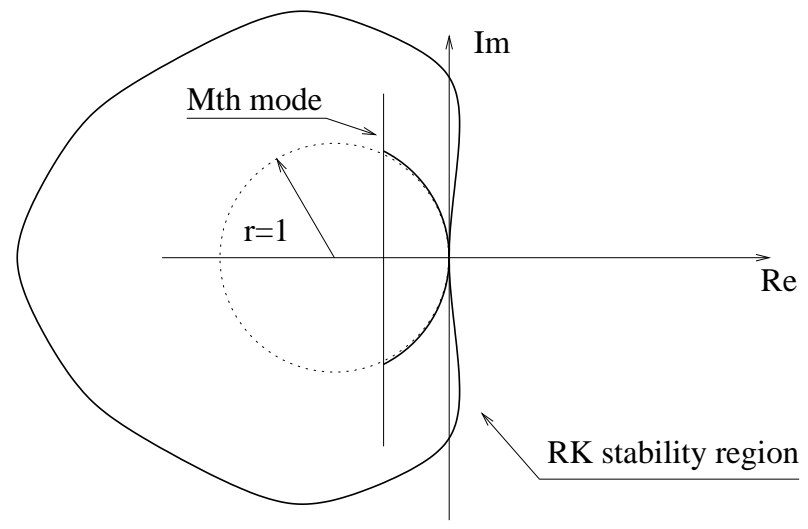

Figure 1: Plane of the eigenvalues of $\widetilde{A} A$. In this case $A$ is the upwind operator. For an explicit time integration the CFL bounds are increased.

where $h$ is the mesh size. The $m$ th eigenvector of matrix $A$ is then given by its components

$$
e^{m}=\left\{e_{j}\right\}^{m}=\alpha^{j} \sin \left(j \theta_{m}\right) ; \quad \alpha=\sqrt{\frac{-\nu / h^{2}-c / 2 h}{-\nu / h^{2}+c / 2 h}}
$$

and where $\theta_{m}=m \pi /(N+1)$ is the frequency parameter, $N$ is the number of discretization points and $j=1 \ldots N$. These eigenvectors approximate the eigenfunctions of the continuous spatial operator with Dirichlet boundary conditions, i.e., $\exp (c x / 2 \nu) \sin (m \pi x)$. However, the eigenfunctions of the continuous spatial operator as well as their discrete approximations are orthogonal only if an appropriate weighted scalar product is used. In the discrete case the weight is $\alpha^{-2 j}$ and we have for example

$$
\left(e^{m}, e^{n}\right)=\sum_{j} e_{j}^{m} e_{j}^{n} \alpha^{-2 j}=\delta_{m n}
$$

Similarly, if the POD basis functions are to recover the advantageous decoupling of eq. 12 , it is necessary that in the definition of eq. 2, the scalar products are defined accordingly using the same weighting function as the continuous operator, i.e., $\exp (-c x / 4 \nu)$ or its discrete counterpart. In this way, the POD basis functions can be found to be the discrete approximations of the eigenfunctions of the continuous operator, so that matrices $\widetilde{A}$ and $A$ commute all the same as in the previous example.

In this linear case we see that the use of a more sophisticated euclidean norm induced by the discrete operator $A$, dramatically improves the approximation properties of the basis functions found by eq. 2. We show in the next Section an attempt to extend such ideas to the compressible Navier-Stokes equations.

$\mathrm{RR} \mathrm{n}^{\circ} 3589$ 
Let us conclude by a non linear example. Consider the case of the inviscid Burgers' equation

$$
u_{t}+u u_{x}=0
$$

with $u=\sum_{k=1}^{M} w^{k}(t) \phi^{k}(x)$. Projecting onto $\phi^{j}(x)$ yields

$$
w_{t}^{j}+\sum_{k=1}^{M} w^{k} w^{j}\left(\phi_{x}^{k}, \phi^{j}\right)=0
$$

Alternatively, using POD as a filter one obtains

$$
U_{t}+\widetilde{A} A(U) U=0
$$

In eq. 17 the term $\left(\phi_{x}^{k}, \phi^{j}\right)$ can be computed once for all, so that the solution of this system of ODEs is very efficient. In this specific case, assuming an implicit time integration the system is stable. Yet, if we proceed in the same way for the Euler [7] and the compressible NavierStokes equations [8] the resulting system of ODEs is unstable. The numerical stabilization of these ODEs systems is still an open question, although in [8] several interesting experiments were performed adding artificial diffusion.

The POD-filter approach is the dual of the constant coefficients ODEs system. It is easily implemented and stable but inefficient. Nevertheless it shows that low order modeling does not necessarily imply instability, and it provides a simple way to study the quality of the approximation which can be obtained by a low order POD-Galerkin formulation.

\section{Compressible flow simulations}

With reference to eq. 1 , we take $W=\sum_{j=1}^{M} w^{j}(t) \phi^{j}(x)$ where $w^{j}(t) \in L^{2}(R)$. In the case of the Euler and laminar Navier-Stokes equations, $\phi^{j} \in\left(L^{2}\left(R^{2}\right)\right)^{4}$, whereas for turbulent flows modeled using the Reynolds averaged Navier-Stokes equations, we have $\phi^{j} \in\left(L^{2}\left(R^{2}\right)\right)^{6}$ in order to account for $\kappa$ and $\varepsilon$. In all of the computations we took $\operatorname{Pr}=0.72$ and $\gamma=7 / 5$. The code on which we base our analysis and computational results combines the following features: a mixed finite volume/finite element formulation on unstructured triangular meshes; the convective part of the Euler equations is discretized using an upwind finite volume formulation based on an approximate Riemann solver [10]. Second order spatial accuracy is obtained through a MUSCL [11] technique which requires the construction of local solution slopes; the latter are being computed using a classical finite element formulation for P1 triangular elements. Linearized implicit formulation for second-order time integration using a defect correction approach [9] is applied. For turbulent flows we resort to a $\kappa-\varepsilon$ model with wall laws.. For the details concerning the test cases that we will present, we refer the reader to [12] and [13]. They were selected as representatives of typical unsteady flows which may be interesting to control.

$P O D$ filter. Based on the code we described above, we use the POD filter constructed in a way similar to that explained in previous Sections. At each time step we compute the

INRIA 
flow variables update using the reference code, then we filter and start the process all over as explained in fig. 2. The POD basis function are obtained by the "snapshots method".

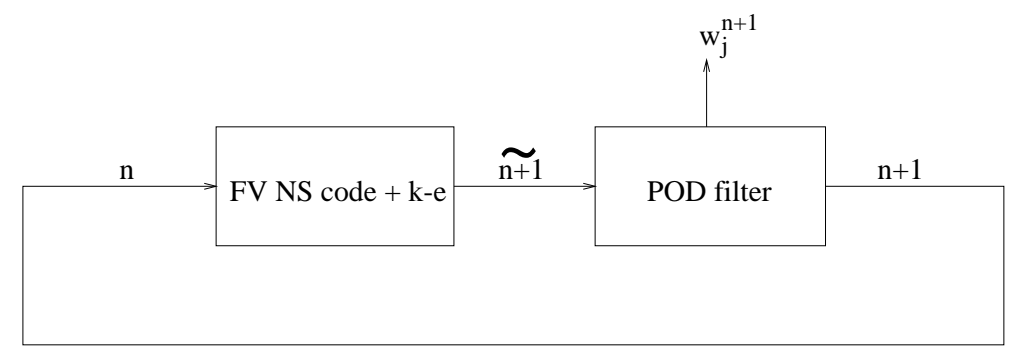

Figure 2: Time integration scheme with POD filter. $\widetilde{A}=\left\{\tilde{a}_{n i}\right\}=\left\{\sum_{k=1}^{M} \bar{\phi}^{k}\left(x_{n}\right) \phi^{k}\left(x_{i}\right)\right\}$

The first case we consider is a NACA0012 at $20^{\circ}$ of incidence immersed in a flow at Mach $=0.2$ and Reynolds $=2100$. The flow field is discretized over a triangular mesh of 12248 vertices. The flow over the airfoil is laminar with periodic vortex shedding from the leading edge and from the trailing edge, see fig. 3. We sample such flow with about 100 snapshots over one period. Then we construct the basis functions and retain 30 of them, those corresponding to the highest eigenvalues. In fig. 4 we see the first component of the second to fifth basis function. Notice that the first basis function is not shown as in our case it is related to the average flow. It is seen that a phase shift appears between the second and third basis functions as well as between the fourth and fifth, whereas the length scale of the structures is constant from the first to the last ones. It may be noticed that the vortex street past the airfoil is out of phase in the first couple of basis functions and in phase in the latter. Indeed, also the corresponding eigenvalues exhibit such a pairing. The length scale of the spatial structures decreases with the subsequent basis functions, not shown here. These facts are very similar to what happens for trigonometric expansions, and we ascribe this to the orthogonality condition.

In fig. 5 we compare the results of the reference code to those obtained using the POD filter. The results are given in terms of lift versus time; there is a slight increase in the oscillation amplitude, which stays however bounded for all times, more remarkable as the number of basis functions used is decreasing as it is seen in fig. 6. The lift plateau due to the vortex shedding from the trailing edge is in all cases except the last over-estimated. The quality of the simulation is reasonable when 10 basis functions or more are used. The explicit reference code, which realizes the time integration with a classic four stage RungeKutta method, operates with a maximum CFL of about 2.5, whereas in the context of the POD filter code the same Runge-Kutta routine can be stably operated with a maximum CFL of about 75 , see fig. 6 . It is remarkable that in accordance to the one-dimensional linear analysis the POD filter code has a stability domain of larger extent.

Constant coefficients system of ODEs. Consider the Navier-Stokes equations rewritten in terms of the primitive variables $\{\varsigma, u, v, p\}$, where $\varsigma=1 / \rho$. Other more usual forms of 


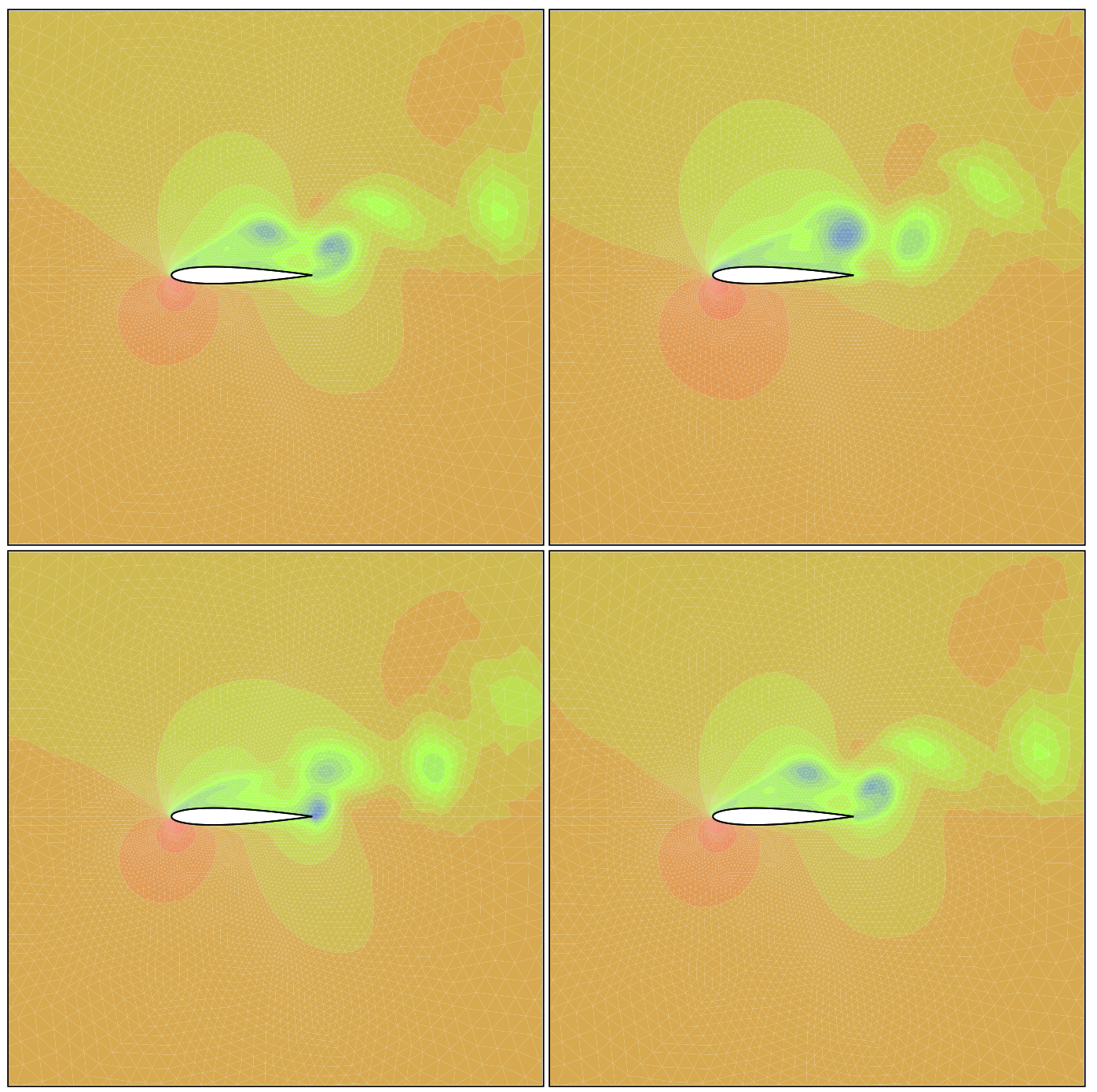

Figure 3: NACA $0012-20^{\circ}$ of incidence, Mach $=0.2, \mathrm{Re}=2100$, laminar. Example of snapshots: density

these equations introduce rational fractions of the unknowns leading to a system of ODEs which is not polynomial, as for example in [8]. We have

$$
\varsigma_{t}+u v_{x}-u_{x} \varsigma-v_{y} \varsigma+v \varsigma_{y}=0
$$




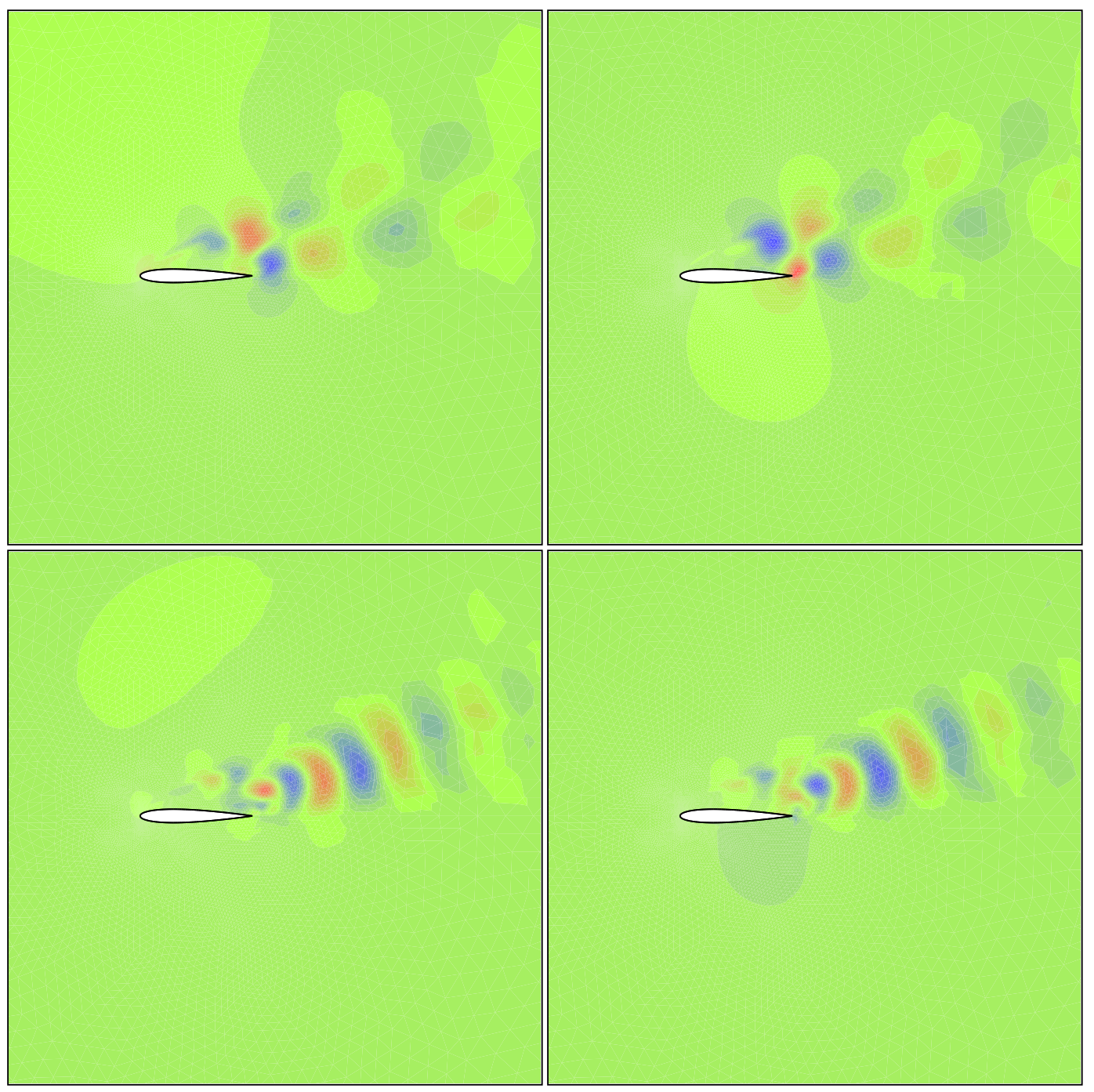

Figure 4: Example of basis functions for the laminar case: first component

$$
\begin{aligned}
& u_{t}+u u_{x}+v u_{y}+\varsigma p_{x}=\frac{1}{R e} \varsigma\left[\left(\frac{4}{3} u_{x}-\frac{2}{3} v_{y}\right)_{x}+\left(v_{x}+u_{y}\right)_{y}\right] \\
& v_{t}+u v_{x}+v v_{y}+\varsigma p_{y}=\frac{1}{R e} \varsigma\left[\left(\frac{4}{3} v_{y}-\frac{2}{3} u_{x}\right)_{y}+\left(v_{x}+u_{y}\right)_{x}\right]
\end{aligned}
$$

$\mathrm{RR} \mathrm{n}^{\circ} 3589$ 


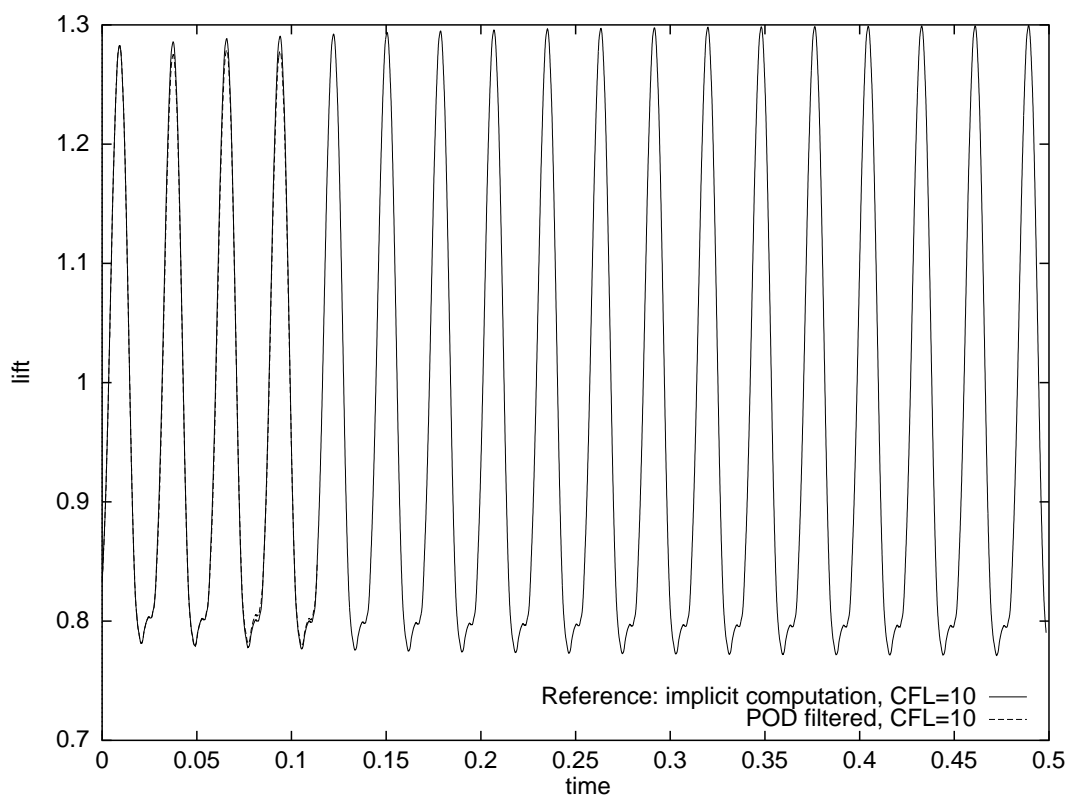

Figure 5: Results of the reference code compared to the ones obtained by POD filtering in the laminar case. The reference computation was pursued for only 4 periods 


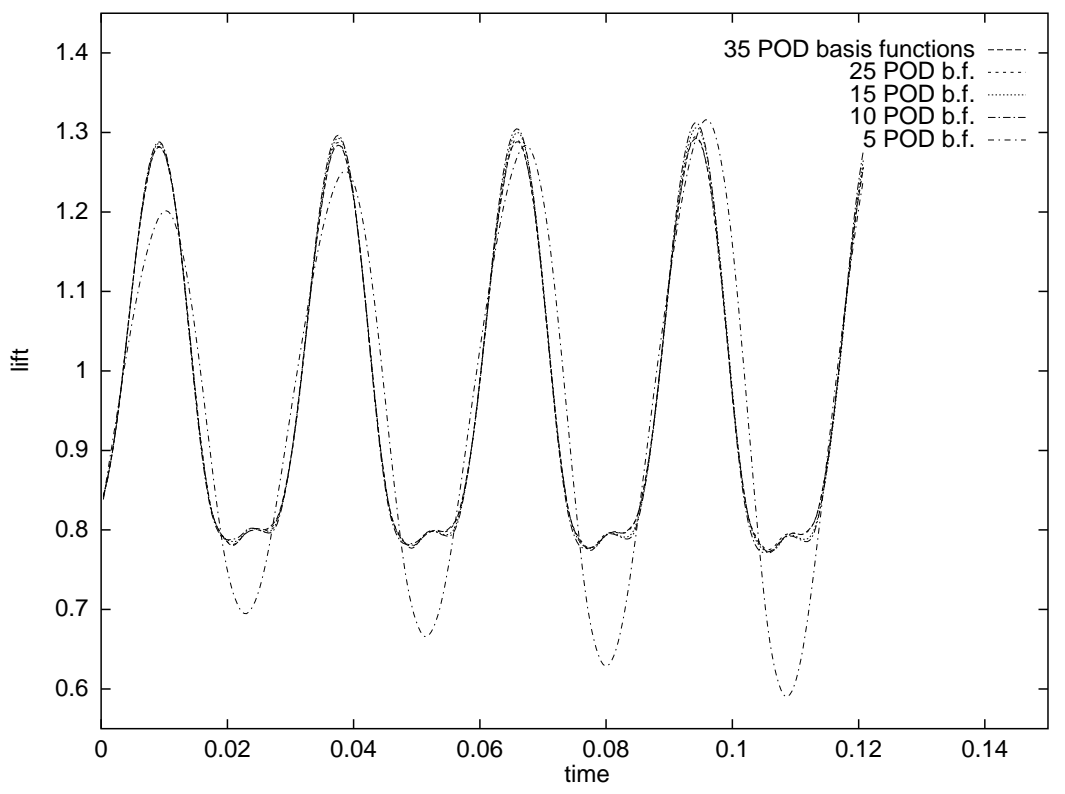

Figure 6: Comparison between the results obtained using the reference code and the ones obtained with different number of POD basis functions at $\mathrm{CFL}=75$.

$\mathrm{RR} \mathrm{n}^{\circ} 3589$ 


$$
\begin{aligned}
& p_{t}+u p_{x}+v p_{y}+\gamma p\left(v_{x}+u_{y}\right)=\frac{\gamma}{\operatorname{Re} \operatorname{Pr}}\left[(p \varsigma)_{x x}+(p \varsigma)_{y y}\right]+ \\
& +\frac{\gamma-1}{\operatorname{Re}}\left[u_{x}\left(\frac{4}{3} u_{x}-\frac{2}{3} v_{y}\right)+v_{y}\left(\frac{4}{3} v_{y}-\frac{2}{3} u_{x}\right)+\left(u_{y}+v_{x}\right)^{2}\right]
\end{aligned}
$$

After projection over $\left\{\phi_{1}^{i}, \phi_{2}^{i}, \phi_{3}^{i}, \phi_{4}^{i}\right\}$, integrating by parts and discarding negligible boundary terms, we obtain a system of the form

$$
\dot{w}_{i}+\sum_{j, k=1}^{M} a_{i j k} w_{j} w_{k}+\frac{1}{R e} \sum_{j, k=1}^{M} b_{i j k} w_{j} w_{k}=0
$$

where

$$
\begin{aligned}
a_{i j k} & =\int_{\Omega}\left(\phi_{1}^{i} \phi_{2}^{j} \frac{\partial \phi_{1}^{k}}{\partial x}+\phi_{1}^{i} \phi_{3}^{j} \frac{\partial \phi_{1}^{k}}{\partial y}-\phi_{1}^{i} \phi_{1}^{k} \frac{\partial \phi_{2}^{j}}{\partial x}-\phi_{1}^{i} \phi_{1}^{k} \frac{\partial \phi_{3}^{j}}{\partial y}\right) d \Omega+ \\
& +\int_{\Omega}\left(\phi_{2}^{i} \phi_{2}^{j} \frac{\partial \phi_{2}^{k}}{\partial x}+\phi_{2}^{i} \phi_{3}^{j} \frac{\partial \phi_{2}^{k}}{\partial y}+\phi_{2}^{i} \phi_{1}^{j} \frac{\partial \phi_{4}^{k}}{\partial x}\right) d \Omega+ \\
& +\int_{\Omega}\left(\phi_{3}^{i} \phi_{2}^{j} \frac{\partial \phi_{3}^{k}}{\partial x}+\phi_{3}^{i} \phi_{3}^{j} \frac{\partial \phi_{3}^{k}}{\partial y}+\phi_{3}^{i} \phi_{1}^{j} \frac{\partial \phi_{4}^{k}}{\partial y}\right) d \Omega+ \\
& +\int_{\Omega}\left(\phi_{4}^{i} \phi_{2}^{j} \frac{\partial \phi_{4}^{k}}{\partial x}+\phi_{4}^{i} \phi_{3}^{j} \frac{\partial \phi_{4}^{k}}{\partial y}+\gamma \phi_{4}^{i} \phi_{4}^{j} \frac{\partial \phi_{2}^{k}}{\partial x}+\gamma \phi_{4}^{i} \phi_{4}^{j} \frac{\partial \phi_{3}^{k}}{\partial y}\right) d \Omega
\end{aligned}
$$

and

$$
\begin{aligned}
b_{i j k} & =\int_{\Omega}\left(\frac{4}{3} \frac{\partial \phi_{2}^{i}}{\partial x} \phi_{1}^{j} \frac{\partial \phi_{2}^{k}}{\partial x}-\frac{2}{3} \frac{\partial \phi_{2}^{i}}{\partial x} \phi_{1}^{j} \frac{\partial \phi_{3}^{k}}{\partial y}\right) d \Omega+ \\
& +\int_{\Omega}\left(\frac{\partial \phi_{3}^{i}}{\partial x} \phi_{1}^{j} \frac{\partial \phi_{2}^{k}}{\partial y}+\frac{\partial \phi_{3}^{i}}{\partial x} \phi_{1}^{j} \frac{\partial \phi_{3}^{k}}{\partial x}\right) d \Omega+ \\
& +\frac{\gamma}{\operatorname{Pr}} \int_{\Omega}\left(\frac{\partial \phi_{4}^{i}}{\partial x} \phi_{1}^{j} \frac{\partial \phi_{4}^{k}}{\partial x}+\frac{\partial \phi_{4}^{i}}{\partial x} \phi_{4}^{k} \frac{\partial \phi_{1}^{j}}{\partial x}\right) d \Omega+ \\
& +\int_{\Omega}\left(\frac{4}{3} \frac{\partial \phi_{3}^{i}}{\partial y} \phi_{1}^{j} \frac{\partial \phi_{3}^{k}}{\partial y}-\frac{2}{3} \frac{\partial \phi_{3}^{i}}{\partial y} \phi_{1}^{j} \frac{\partial \phi_{2}^{k}}{\partial x}\right) d \Omega+ \\
& +\int_{\Omega}\left(\frac{\partial \phi_{2}^{i}}{\partial y} \phi_{1}^{j} \frac{\partial \phi_{2}^{k}}{\partial y}+\frac{\partial \phi_{2}^{i}}{\partial y} \phi_{1}^{j} \frac{\partial \phi_{3}^{k}}{\partial x}\right) d \Omega+ \\
& +\frac{\gamma}{\operatorname{Pr}} \int_{\Omega}\left(\frac{\partial \phi_{4}^{i}}{\partial y} \phi_{1}^{j} \frac{\partial \phi_{4}^{k}}{\partial y}+\frac{\partial \phi_{4}^{i}}{\partial y} \phi_{4}^{k} \frac{\partial \phi_{1}^{j}}{\partial y}\right) d \Omega+ \\
& +(\gamma-1) \int_{\Omega}\left(\frac{4}{3} \frac{\partial \phi_{2}^{j}}{\partial x} \phi_{4}^{i} \frac{\partial \phi_{2}^{k}}{\partial x}-\frac{2}{3} \frac{\partial \phi_{2}^{j}}{\partial x} \phi_{4}^{i} \frac{\partial \phi_{3}^{k}}{\partial y}\right) d \Omega+
\end{aligned}
$$

INRIA 


$$
\begin{aligned}
& +(\gamma-1) \int_{\Omega}\left(\frac{4}{3} \frac{\partial \phi_{3}^{j}}{\partial y} \phi_{4}^{i} \frac{\partial \phi_{3}^{k}}{\partial y}-\frac{2}{3} \frac{\partial \phi_{3}^{j}}{\partial y} \phi_{4}^{i} \frac{\partial \phi_{2}^{k}}{\partial x}\right) d \Omega+ \\
& +\quad(\gamma-1) \int_{\Omega}\left(\frac{\partial \phi_{2}^{j}}{\partial y} \phi_{4}^{i} \frac{\partial \phi_{2}^{k}}{\partial y}+2 \frac{\partial \phi_{3}^{j}}{\partial x} \phi_{4}^{i} \frac{\partial \phi_{2}^{k}}{\partial y}+\frac{\partial \phi_{3}^{j}}{\partial x} \phi_{4}^{i} \frac{\partial \phi_{3}^{k}}{\partial x}\right) d \Omega
\end{aligned}
$$

The coefficients $a_{i j k}$ and $b_{i j k}$ are computed once for all using the basis functions and their gradients computed as in eq. 11. The system of ODEs is integrated in time by the backward differentiation formula for stiff systems of ODEs (Gear method).

In the airfoil test case, the period $(0.028 \mathrm{~s})$ of the oscillations found by numerical integration of eq. 19 is in agreement with that obtained using the reference or the POD filter scheme. Yet, one can see in fig. 7(a) for the coefficients $w_{2}(t)$ and $w_{3}(t)$ that amplitude of the oscillation increases to finally stabilize. In the same figure the phase-space cut on the plane of the coefficients $w_{2}-w_{3}$ and $w_{4}-w_{5}$ is compared to what is obtained by POD filtering. The ordered motion of fig. 7(c) is disrupted in fig. 7(b). We ascribe this behavior to a lack of numerical diffusion. If additional stabilization is provided in a brutal way, i.e. increasing the viscosity, the results of the constant coefficients system are in good agreement with those of the reference numerical simulation, compare fig. 7(c) and fig. 8(b).

We now turn our attention to a turbulent test case: we consider a square cylinder immersed in flow at Mach number 0.1 and Reynolds number 22000. A $\kappa-\varepsilon$ model with wall laws was applied. The flow field is discretized over a triangular mesh with 9274 vertices. The flow computation performed with the reference finite-volumes code shows a periodic vortex shedding from the corners of the square. In order to build the basis functions we took 100 snapshots of the flow over one period; the first component and the third component of $\phi^{2}-\phi^{8}$ are shown in figs. 10 and 9 . We observe an odd-even decoupling of the modes as well as a phase shift, as in the previous case.

The POD filter was constructed with 30 basis functions and the results are shown in fig. 11. Again the low order model is in good agreement with the reference code. The phase space sections for $w_{2}-w_{3}, w_{4}-w_{5}$ and $w_{6}-w_{7}$ are given in fig. 12. It is seen that the motion on this planes is ordered and that the shapes of the closed orbits denote the presence of oscillation with increasing time frequency as the number of basis function increases.

$H^{1}$ formulation. In the POD-filter code we used for the laminar and turbulent flow experiments the numerical diffusion is introduced by solving Riemann problems at control volume interfaces, ensuring sufficient stabilization so that the low order model is also stable. In order to develop a rational method to introduce diffusion in the constant coefficient system of ODEs eq. 19, we explored the possibility of redefining the norms involved in the POD definition of Section 2. Indeed it seems to us that to increase the relevance of smaller scales in the POD definition might enhance dissipation. This can be accomplished for example by redefining all the norms in the Sobolev space $H^{1}$, so that the derivatives of the snapshots as well as that of the basis functions are included in the POD average. The formula to compute the POD basis functions becomes

$$
\phi=\max _{\psi \in H^{1}}<(W, \psi)^{2}>
$$



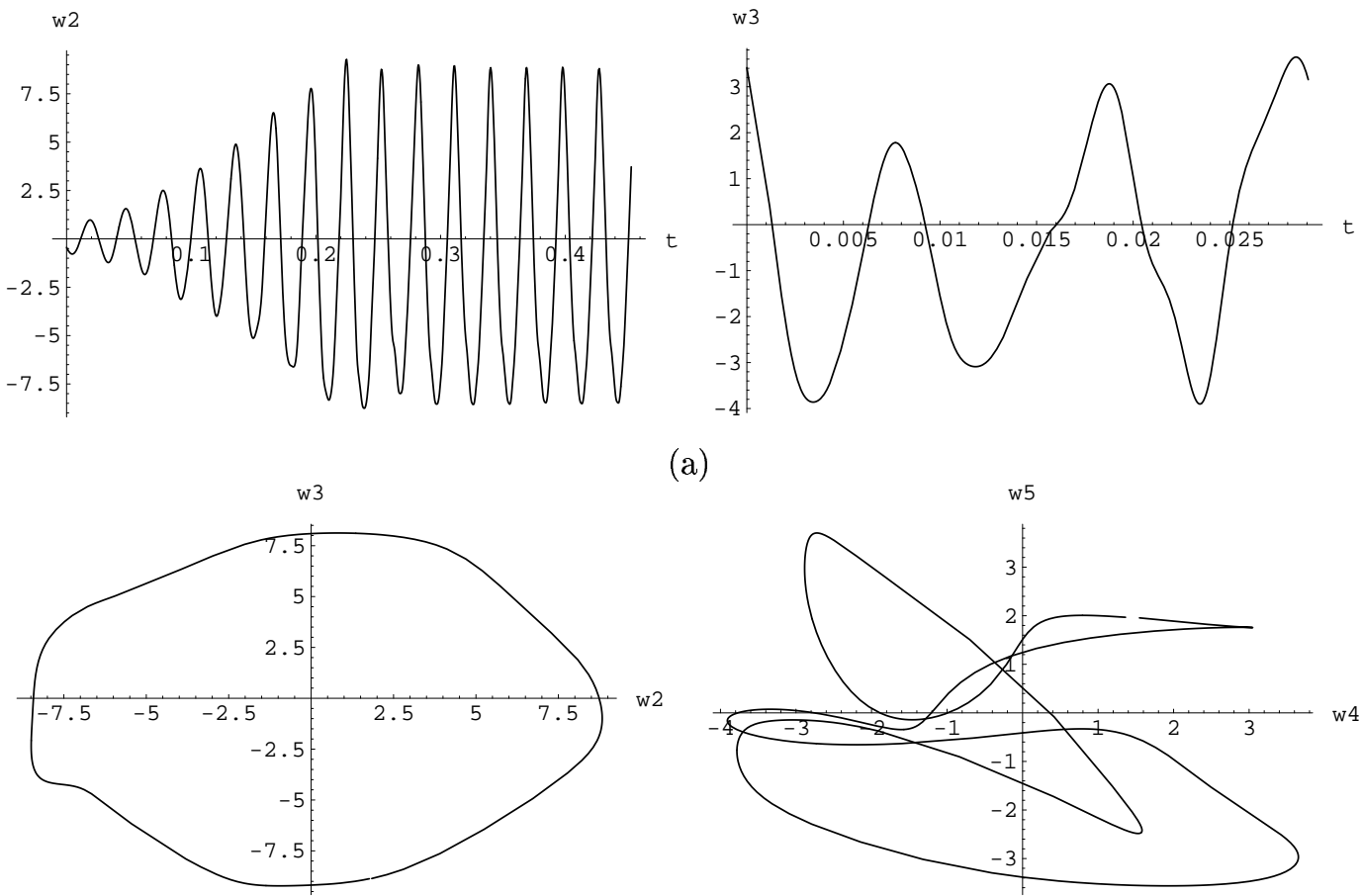

(a)

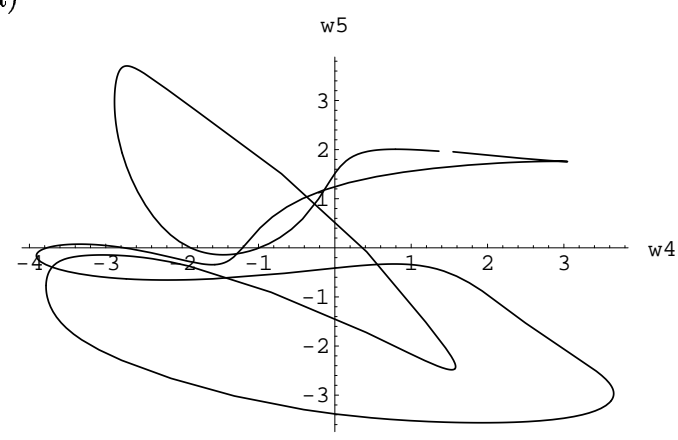

(b)
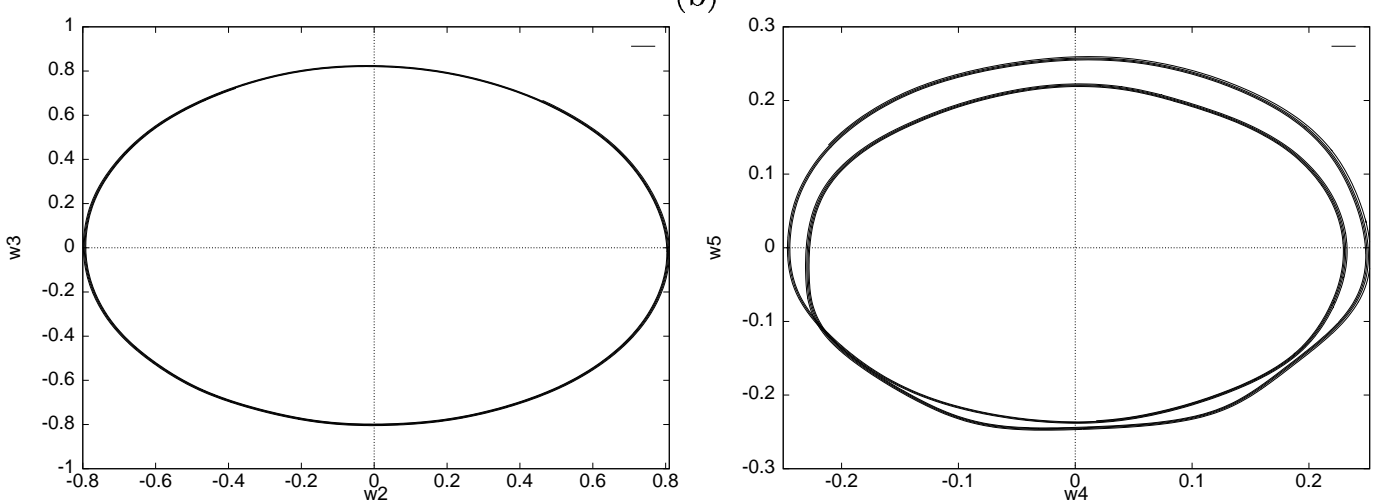

(c)

Figure 7: Constant coefficient system without additional dissipation: (a) second and third coefficient versus time, (b) phase-space sections; projection of the numerical solution obtained by the FV code: (c) phase-space sections. 

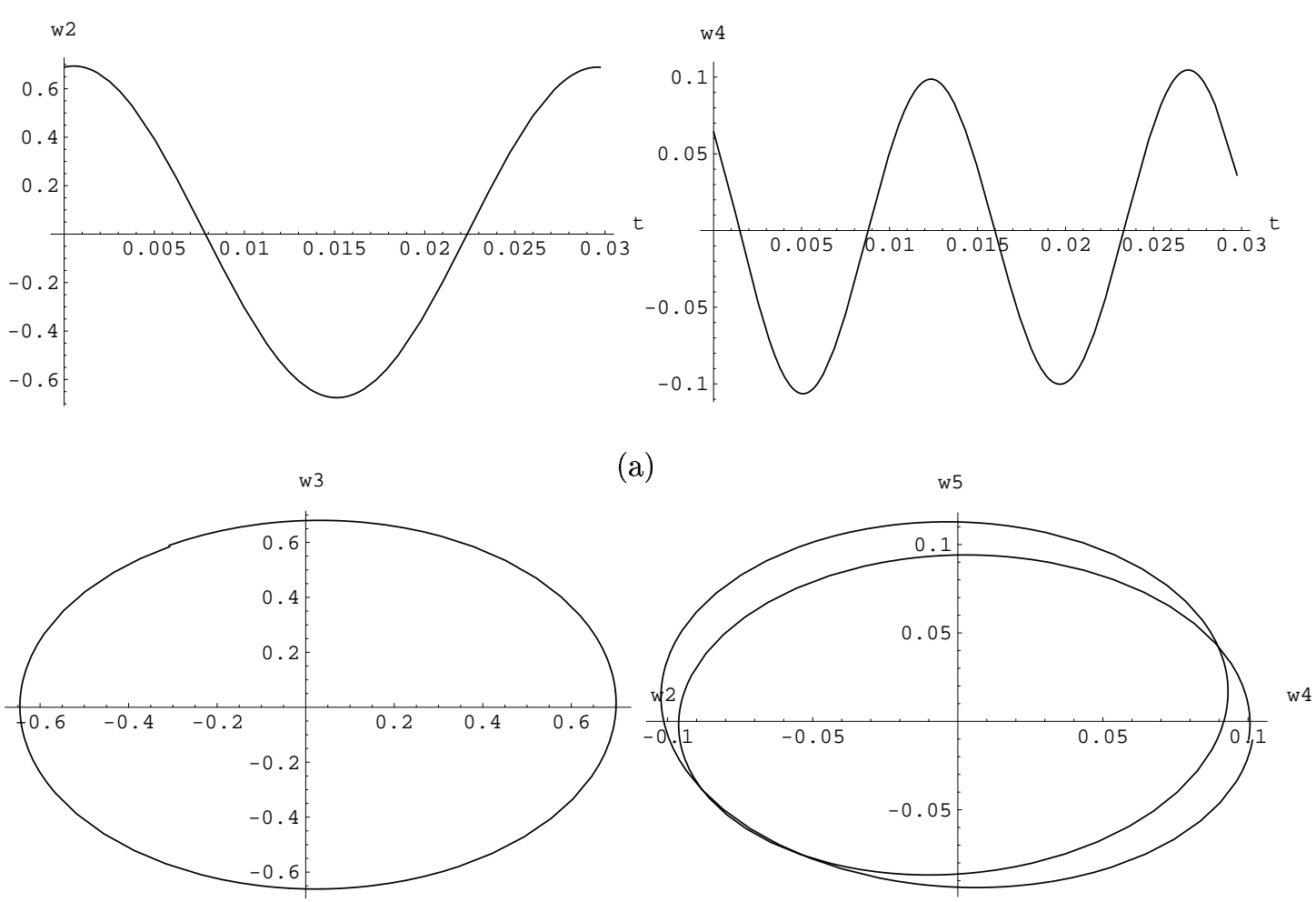

(b)

Figure 8: System with increased viscosity: (a) second and third coefficient versus time, (b) phase-space sections.

$$
=\max _{\psi \in H^{1}}<\left(\int_{\Omega} W \psi d \Omega+\varepsilon \int_{\Omega} \nabla W \cdot \nabla \psi d \Omega\right)^{2}>
$$

Many metrics are possible for $H^{1}$, so that $\varepsilon$ can be conveniently chosen in different simulations. From dimensional analysis considerations, one may guess $\varepsilon$ proportional to $T / R e$, where $T$ is some appropriate time scale. The basis functions are found by linear combination of the solution snapshots as in the $L^{2}$ case, yet the coefficients of the linear combination are now the eigenvectors of the matrix

$$
\left\{c_{n m}\right\}=\left\{\int_{\Omega} W^{(n)} W^{(m)} d \Omega+\varepsilon\left(\int_{\Omega} \nabla W^{(n)} \cdot \nabla W^{(m)} d \Omega\right)\right\}
$$

where the numerical evaluation of the nodal gradient $\nabla W$ is computed as an average over the cell of the P1 gradients. Accordingly, the orthogonality condition is $\left(\phi^{i}, \phi^{j}\right)_{H^{1}}=\delta_{i j}$. 


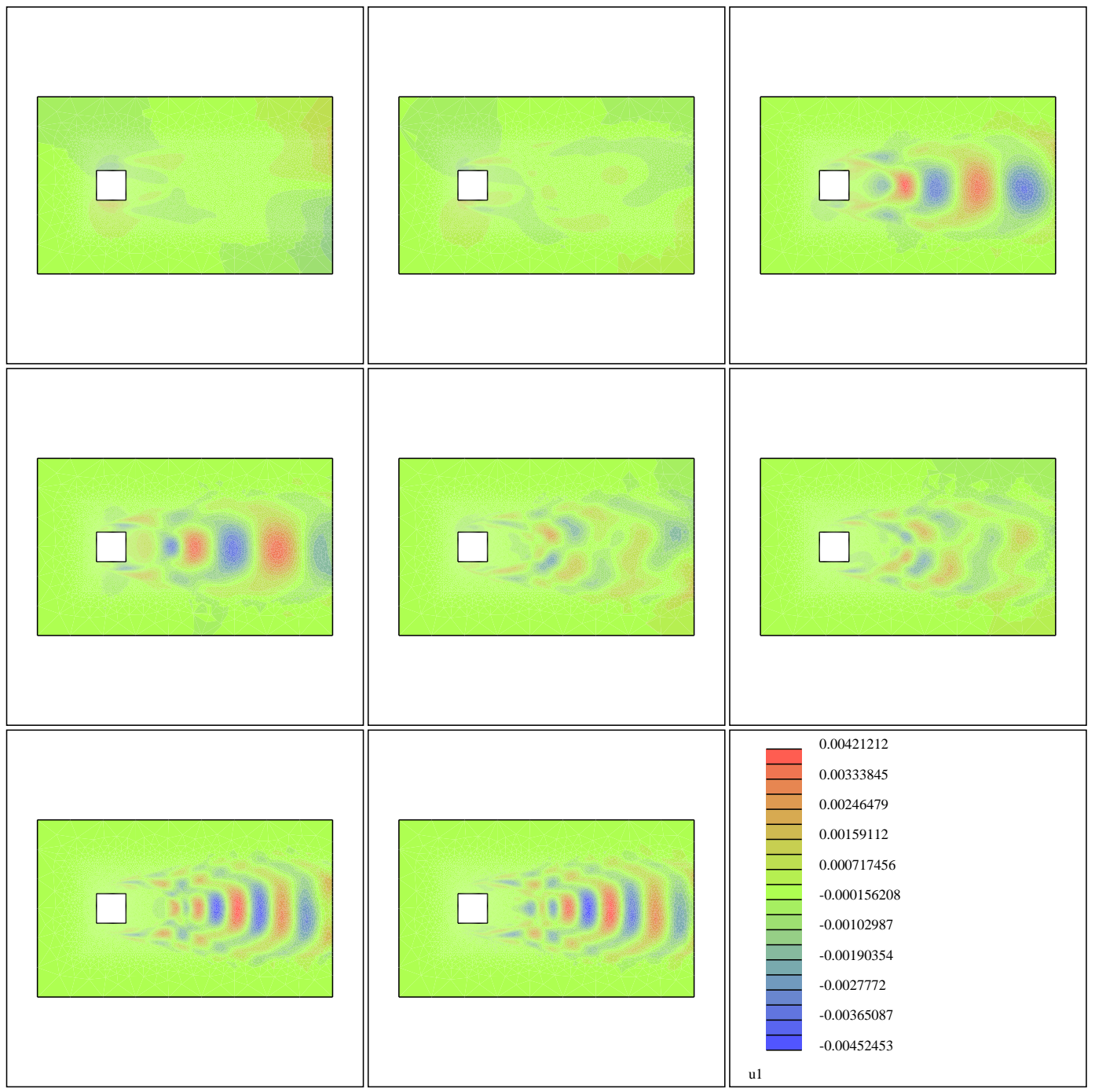

Figure 9: Square cylinder, Mach $=0.1, R e=22000$, turbulent. 100 Snapshots; 10 basis functions. First component of POD basis functions

The numerical simulations which we now present are obtained based on the POD-filter code in which the filter is built using the basis defined in $H^{1}$. Our aim is to investigate the approximation properties of such functions as compared to those obtained with the $L^{2}$ norm. 


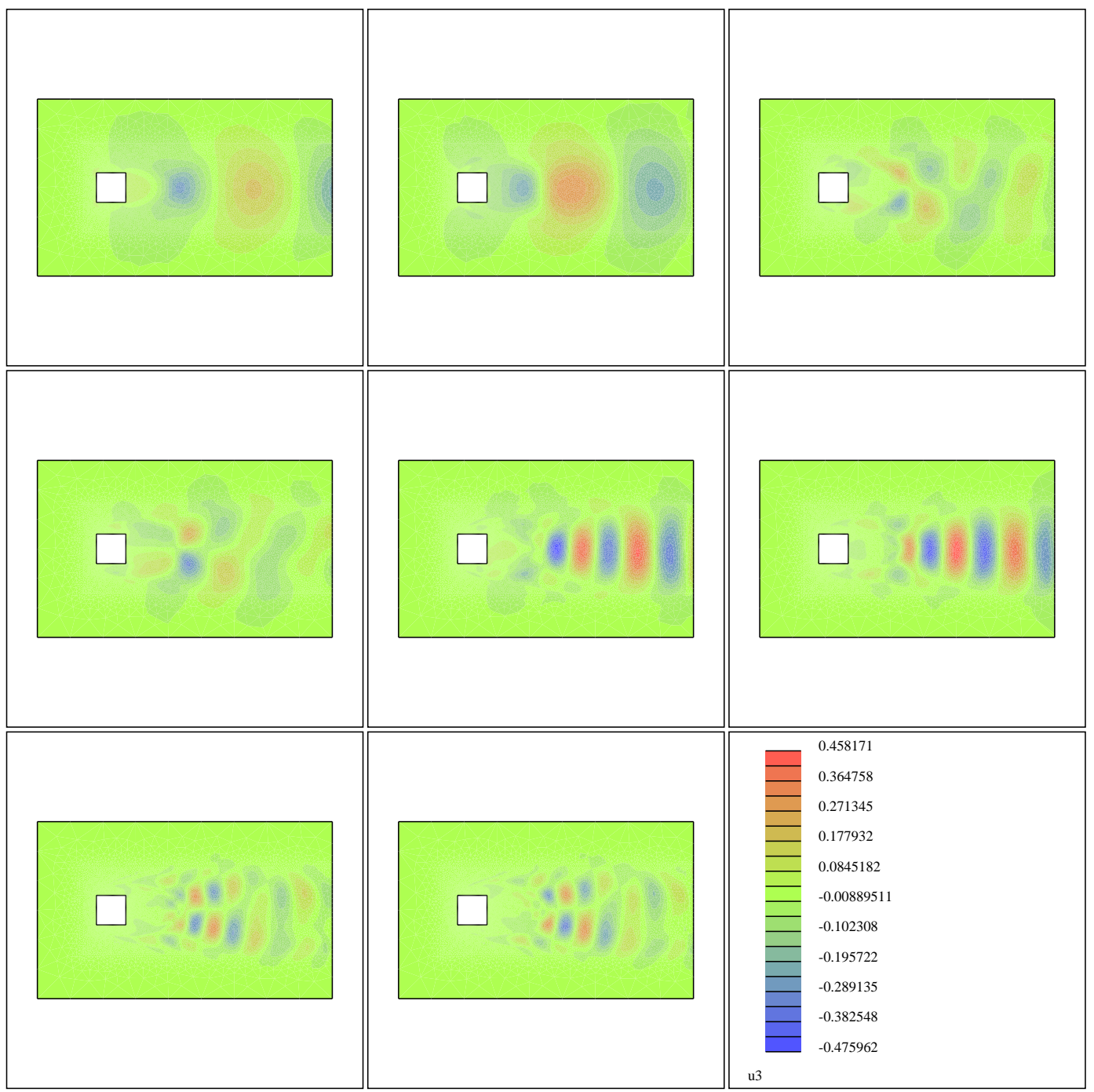

Figure 10: Third component of POD basis functions for the square cylinder case

We expect that as the Reynolds number is increasing, the advantage of the $H^{1}$ formulation over the $L^{2}$ becomes more and more evident. In the laminar test case, which was studied for $R e=2100$, we now double the Reynolds number and compute the flow starting from a uniform initial condition with the basis functions relative to the $R e=2100$ case. This simulation is different from that of fig. 5 , as the transitory solution occurring before 


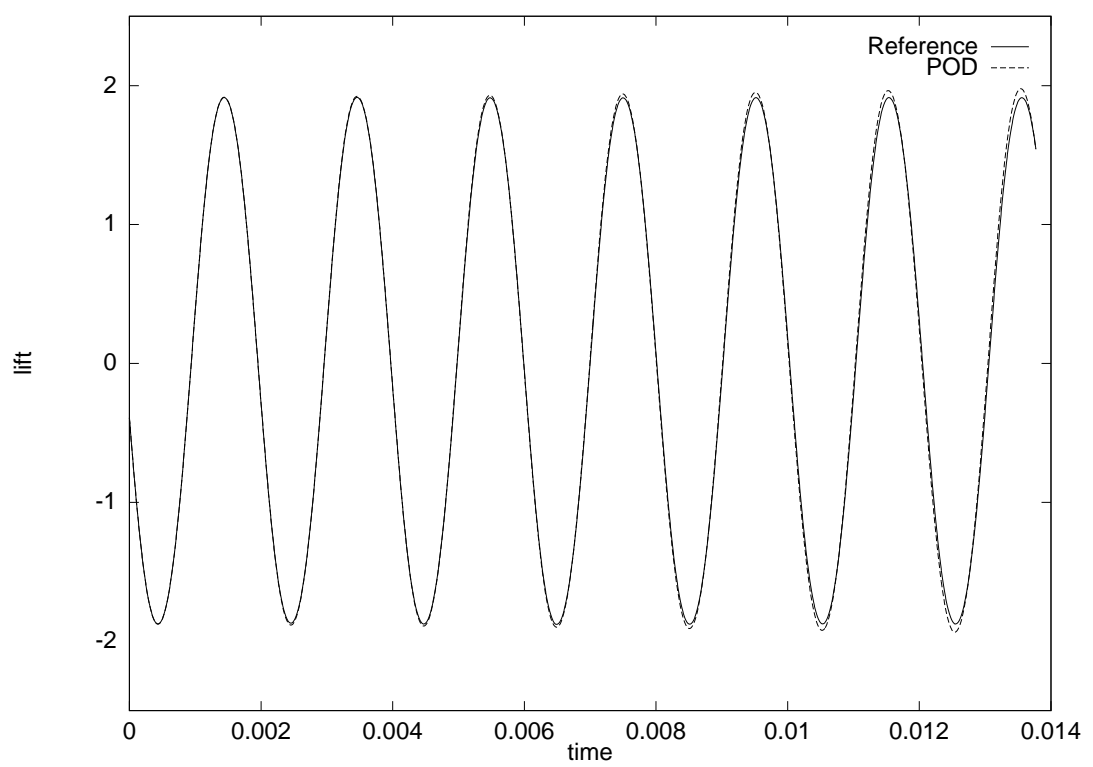

Figure 11: Results of the reference code compared to the ones obtained by POD filtering in the turbulent case. 

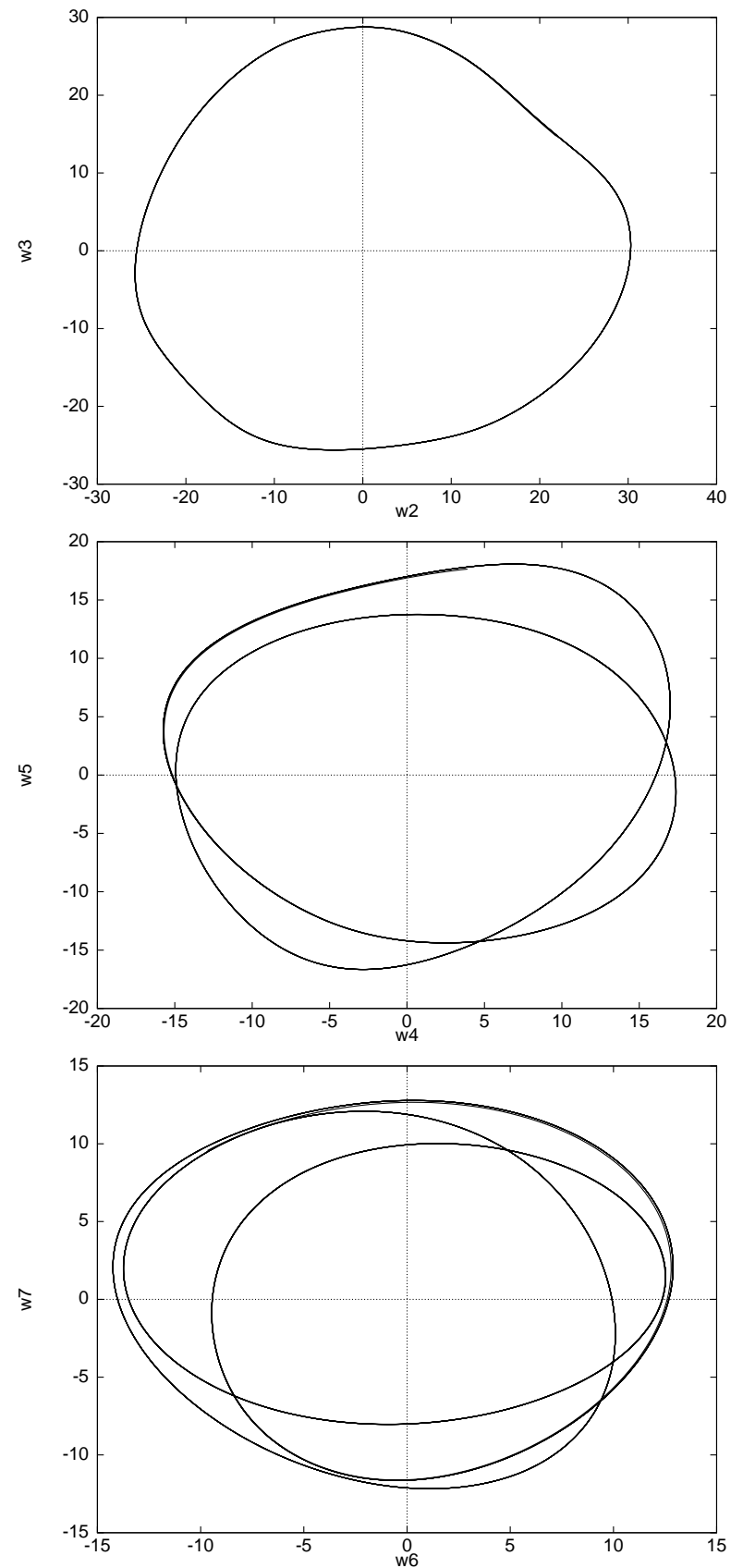

Figure 12: Square cylinder: phase space sections

$\mathrm{RR} \mathrm{n}^{\circ} 3589$ 
the establishment of the periodic vortex shedding does not belong to the data-base from which we derive the POD basis functions. Nevertheless, it is seen in fig. 13(a) that POD basis functions derived with the $H^{1}$ norm are still in good agreement with the reference solution, whereas the the $L^{2}$-norm basis functions are unable to give a reasonable representation of the flow. If we initialize the computation from the established periodic flow, still at $R e=4200$, the solution obtained with the $L^{2}$-norm basis functions compare better with the reference flow, see fig. 13(b). Yet, it seen that the amplitude of the oscillation tends to increase with time.

Indeed also the next example shows that the $H^{1}$-basis functions seem to allow better results even in the case of a turbulent flow. The experimental set up is the following: in the cylinder case $(\mathrm{Re}=22000)$ the convective fluxes are computed using a centered approximation. Then we compare the the $L^{2}$ POD filtered solution and the $H^{1}$ POD filtered solution. The reference code is in this case numerically unstable, as well as the $L^{2}$ POD filter code. On the contrary, the $H^{1}$ formulation with $\varepsilon=10$ and 10 basis function provides sufficient stabilization so that the computation is still in good agreement with the reference computation, as it is seen in fig. 14. Therefore, we observe a definite benefit in employing the $H^{1}$ formulation of the POD.

\section{Conclusions}

We have showed that POD can be viewed as a filtering process. The results based on a POD-filter canonical code are in good agreement with the reference, both in a laminar and a turbulent case. We conclude that the expectation of simulating a complex flow on the basis of a limited number of degrees of freedom is founded. Through the canonical code on which we base our POD approach we are able to provide a rational way to add numerical diffusion to the ODEs system. We have derived the constant coefficients system of ODEs relative to the compressible Navier-Stokes equations, and shown that, however, its judicious numerical stabilization is crucial to retain the approximation properties found using the POD-filter code. Based on numerical experiments, we suggest that one way to achieve stabilization improvements is to define POD in the $H^{1}$ Sobolev space, that is to incorporate gradients as well as function values in the definition of POD. In conclusion the perspectives opened are to devise numerical expedients to include the optimal diffusion amount in the constant system of ODEs relative to the compressible Navier-Stokes equations, taking advantage of the apparent benefits of the $H^{1}$ formulation.

\section{Acknowledgments}

The first author expresses his gratitude to Dr. A. Dervieux (INRIA) for his constant support and encouragement. Particular thanks are due to Dr. R. Peyret (CNRS) for valuable recommendations as well as to Dr. M. Mallet and Mr. G. Vigo (Dassault Aviation) for several fruitful discussions contributing to this work. 


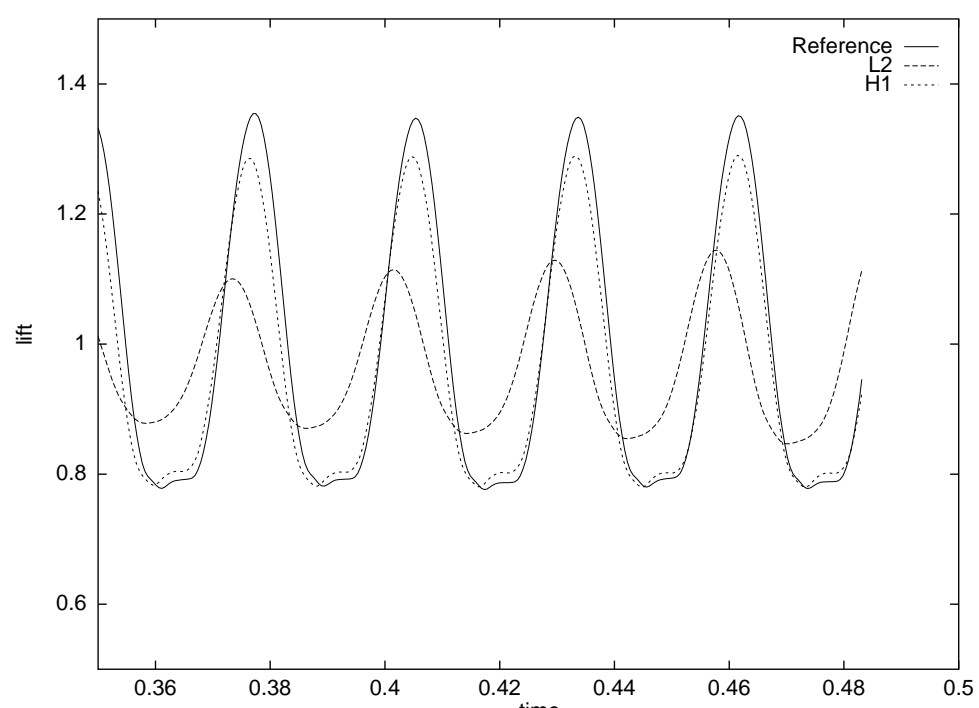

(a) ${ }^{\text {time }}$

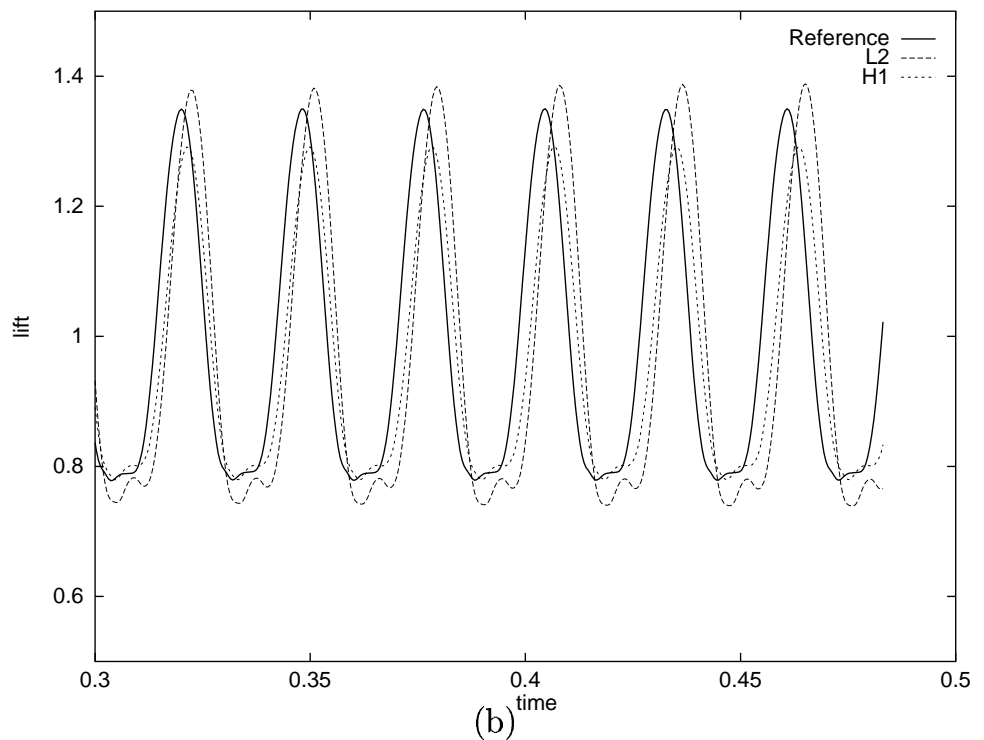

Figure 13: $R e=4200$. Comparison between the results obtained using the reference code and the ones obtained by POD filtering with basis functions relative to $R e=2100$ : (a) uniform flow initial condition; (b) initialization from the established periodic flow.

$\mathrm{RR} \mathrm{n}^{\circ} 3589$ 


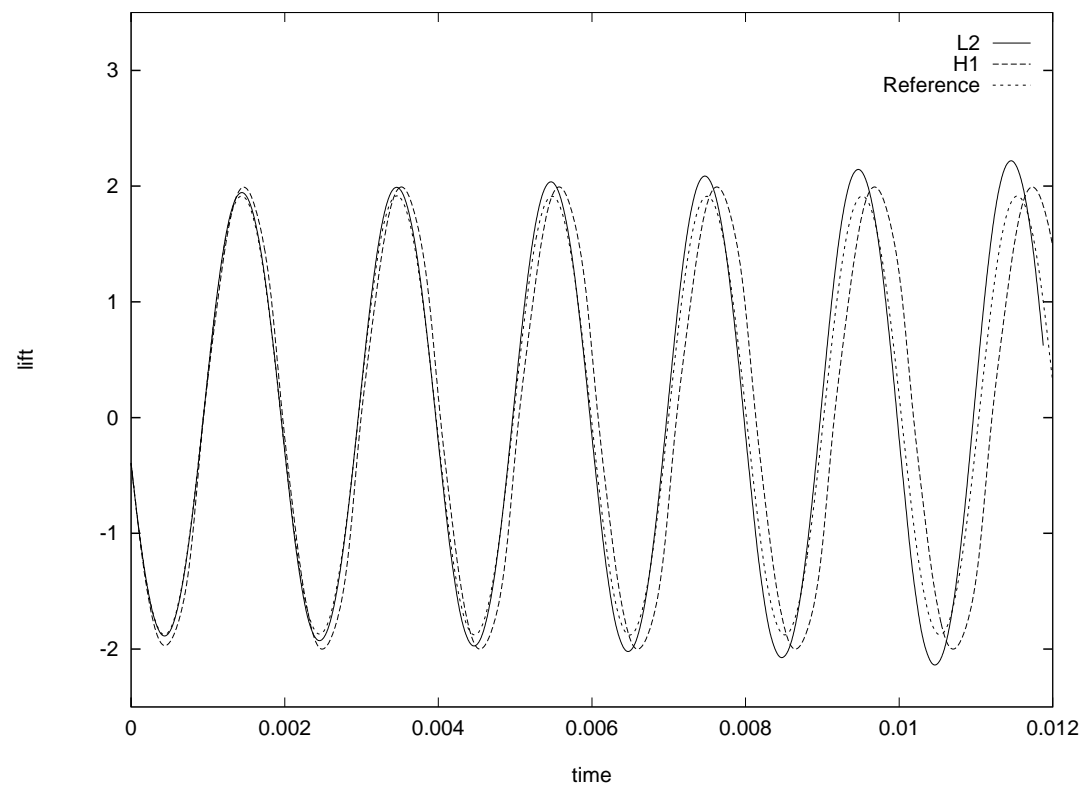

Figure 14: Square cylinder case: reference versus the $L^{2}$ and the $H^{1}$ POD-filter computations.

\section{References}

[1] J.L. Lumley, 1967. The structure of inhomogeneous turbulent flow. In Atmospheric Turbulence and Wave Propagation, eds. A.M. Yaglom and V.I. Tatarski, pp.166-178. Nauka, Moscow.

[2] G. Berkooz, P. Holmes, J.L. Lumley, 1993. The proper orthogonal decomposition in the analysis of turbulent flows. Annual Review of Fluid Mechanics, Vol. 25, pp. 539-575.

[3] L. Sirovich, 1987. Turbulence and the dynamics of coherent structures. Part I: coherent structures. Quarterly of Applied mathematics, Vol. XLV, n. 3, pp. 561-571.

[4] N. Aubry, P. Holmes, J.L. Lumley, E. Stone, 1988. The dynamics of coherent structures in the wall region of a turbulent boundary layer. Journal of Fluid Mechanics, Vol. 192, pp. 115-173.

[5] K.Y. Tang, W.R. Graham, J. Peraire, 1996. Active flow control using a reduced order model and optimum control. AIAA Paper 96-1946.

[6] R. Courant, D. Hilbert, 1953. Methods of mathematical physics. Vol. I. John Wiley \& Sons, New York. 
[7] A. Iollo, Remarks on the approximation of the Euler equations by a low order model. INRIA Research Report RR-3329, 1997.

[8] G. Vigo, The proper orthogonal decomposition applied to unsteady compressible Navier Stokes equations. INRIA Research Report RR-3385, 1998.

[9] R. Martin, H. Guillard, A second order defect correction scheme for unsteady problems, Computer and Fluids, Vol. 25, No. 1, pp. 9-27.

[10] P.L. Roe, Approximate Riemann solvers, parameters vectors and difference schemes, Journal of Computational Physics, Vol. 43, pp. 357-371, 1981.

[11] B. van Leer, Towards the ultimate conservative difference scheme $V:$ a second-order sequel to Godunov's method, Journal of Computational Physics, Vol. 32, pp. 361-370, 1979.

[12] T.H. Pulliam, Low Reynolds number numerical solution of chaotic flows, AIAA Paper 89-0123.

[13] H. Tran, B. Koobus, C. Farhat. Numerical solution of vortex dominated flow problems with moving grids, AIAA Paper 98-0766.

\section{Contents}

1 Introduction $\quad 3$

2 POD of the compressible Navier-Stokes equations 4

3 Analysis of POD applied to model equations $\quad 7$

4 Compressible flow simulations $\quad 10$

5 Conclusions $\quad 24$

$\mathrm{RR} \mathrm{n}^{\circ} 3589$ 


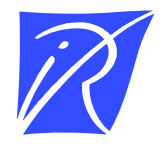

Unité de recherche INRIA Sophia Antipolis 2004, route des Lucioles - B.P. 93 - 06902 Sophia Antipolis Cedex (France)

Unité de recherche INRIA Lorraine : Technopôle de Nancy-Brabois - Campus scientifique 615, rue du Jardin Botanique - B.P. 101 - 54602 Villers lès Nancy Cedex (France)

Unité de recherche INRIA Rennes : IRISA, Campus universitaire de Beaulieu - 35042 Rennes Cedex (France)

Unité de recherche INRIA Rhône-Alpes : 655, avenue de l'Europe - 38330 Montbonnot St Martin (France)

Unité de recherche INRIA Rocquencourt : Domaine de Voluceau - Rocquencourt - B.P. 105 - 78153 Le Chesnay Cedex (France)

Éditeur

INRIA - Domaine de Voluceau - Rocquencourt, B.P. 105 - 78153 Le Chesnay Cedex (France)

http://www.inria.fr

ISSN 0249-6399 